\title{
Field Cycle Length Sweep to Evaluate Resonant Cycle Sensitivity
}

by

Howell Li

Purdue University

Christopher M. Day

Purdue University

Alexander M. Hainen

University of Alabama

Amanda Stevens

Indiana Department of Transportation

Steven M. Lavrenz

Purdue University

W. Benjamin Smith

Indiana Department of Transportation

Hayley Summers

Purdue University

Richard S. Freije

Indiana Department of Transportation

James R. Sturdevant

Indiana Department of Transportation

Corresponding author:

Darcy M. Bullock

Purdue University

400 Centennial Mall Drive

West Lafayette, IN 47906

Phone (765) 494-2226

darcy@purdue.edu

October 7, 2014 


\begin{abstract}
Cycle length selection in corridor timing is often dictated by critical intersections with the highest level of saturation. Along corridors with balanced volumes and favorable link distances, a resonant cycle length is often sought to provide good progression in both directions of travel. This paper discusses the search for a resonant cycle length at a 5-intersection corridor in Fishers, Indiana over a three month period. The software traffic model suggests a reasonable range of cycle lengths from 104 seconds to 124 seconds for the corridor. This cycle length range is consistent with analytical highway capacity manual delay minimization approaches.

A set of eleven cycle lengths from the 104 to 124-second range are tested over 12 weeks, with each iteration using optimized offset values generated by the Link Pivot progression optimization algorithm to maximize the percentage of vehicles arriving on green, and holding all phase splits constant. There was no obvious resonant cycle identified in the cycle length sweep, however the experiment findings indicate vehicles arriving on green decrease and travel times increase as cycle length increases. As a tradeoff, the number of force off phase terminations on the side-street phases decrease as a result of longer cycles indicating a better accommodation of sidestreet demand. Finally, a Seemingly Unrelated Regression (SURE) model was used to analyze the correlation between cycle lengths, percent of vehicles arriving on green, and travel time indicating a negative correlation between higher cycle length and progression performance.
\end{abstract}

\title{
INTRODUCTION
}

Although a bit of an oversimplification, one could argue that arterial signal systems have two objectives. One is to allocate capacity among conflicting movements, and another is to progress traffic. When considering any aspect of signal operations, two perspectives are possible: (1) capacity allocation, and (2) progression.

Cycle length is used in coordinated signal control to synchronize operations at neighboring intersections in order to progress traffic. Selection of cycle length is often the first task in establishing a signal timing plan for a given time of day. The two perspectives on signal operations lead to two strategies for selecting cycle length.

From the capacity allocation perspective, one strategy is to use the value needed by the intersection with the heaviest demand, seeking a maximum degree of saturation of $90 \%$ [1]. This type of strategy is used to adjust cycle lengths in SCOOT [2] and VFC-OPAC [3]. In these systems, when the intersection degree of saturation reaches $90 \%$, the cycle length is incrementally increased. When the degree of saturation falls substantially beneath this value, the cycle length may be incrementally reduced. Similar strategies are used in offline optimization methods.

From the progression perspective, another strategy is to seek a resonant cycle length, which should be equal to integer multiples of twice the travel time on the segments between neighboring intersections. This strategy is reasonable when traffic is balanced in both directions along the 
arterial, as it uses the symmetric characteristics to establish two-way progression. Cycle length resonance was observed by Koshi [4] in a study of online feedback control of cycle length. Shelby et al. [1] found evidence for resonant cycle length in simulation experiments in the presence of plateaus in the cycle length-performance index curves obtained from modeling in TRANSYT-7F. To date, there has been one subsequent study [5] on resonant cycle length, which was a simulation study. A resonant cycle was not found in that study network.

In order to develop improved systems for providing feedback on signal operations, a study was carried out in Indiana to empirically determine the impact of cycle length on coordinated operations. The cycle length of a coordinated arterial was varied across a range of values in which a resonant cycle was expected to be found. The impacts of cycle length were measured from highresolution signal event data and measurements of travel time. This paper presents the outcomes of that study.

\section{MODEL-BASED OPTIMAL CYCLE LENGTHS}

Figure 1 shows a map of the test network considered in this study. This is State Road 37 in Fishers, Indiana. The system comprises five intersections, and has a speed limit of $55 \mathrm{mph}$. The five intersections have approximately regular spacing, with the location of the middle intersection $\left(135^{\text {th }} \mathrm{Pl}\right.$.) offset from the system midpoint by 5 seconds. In this study, operation during the early evening (19:00-22:00) was investigated.

To determine whether a resonant cycle would be expected to exist in this system, a software model of the network was created in Synchro [6] and populated with representative volumes on each movement. The manual cycle length optimization procedure was then carried out to obtain performance measures from each cycle length within a wide range. Figure 2 a shows a cycle length sweep plot of the Performance Index (PI). The PI is equal to [6]:

$$
P I=\frac{D+S * 10}{3600}
$$

Equation 1

where $D$ is the total delay and $S$ is the number of stops.

Figure $2 \mathrm{a}$ indicates that the minimal PI occurs at a cycle length of 112 seconds, though there is a wider valley of feasible values around the minimum. Based on these results, it was decided that the range of 104 to 124 seconds would be feasible for an empirical cycle length evaluation. Cycle lengths beneath 104 seconds would provide inadequate green times on certain movements, while cycle lengths greater than 124 seconds would result in excessive queues. 


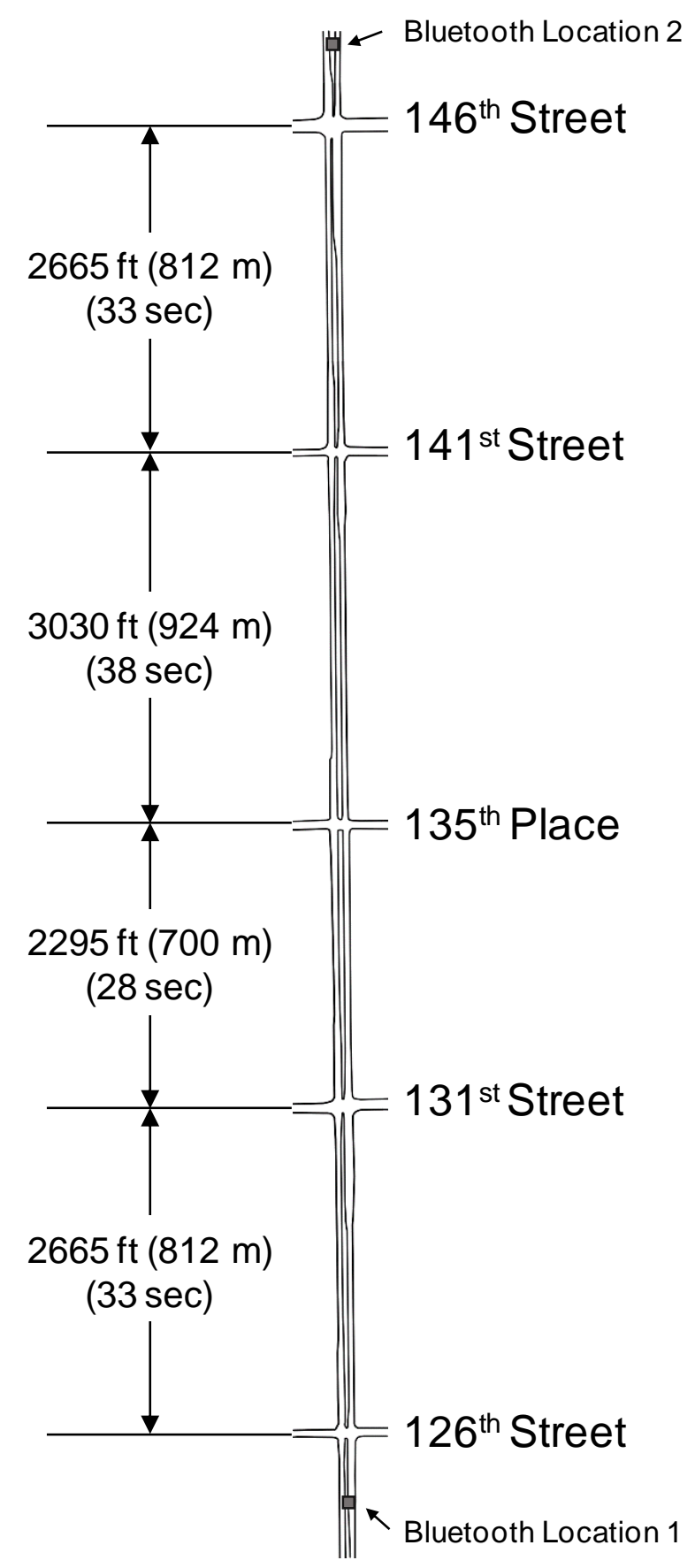

Figure 1. Indiana State Road 37 (Fishers, Indiana). 


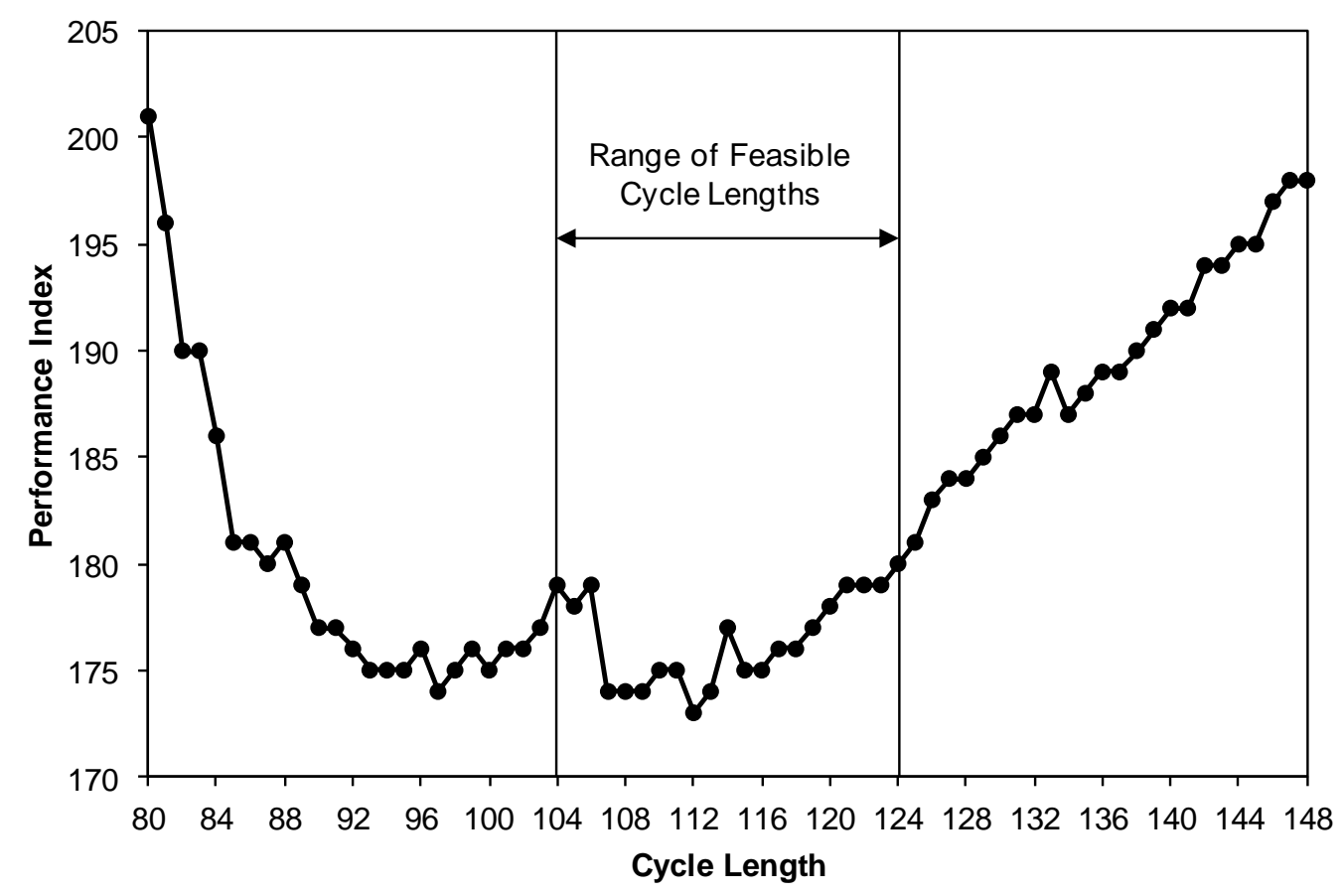

(a) Progression: Results of optimization software.

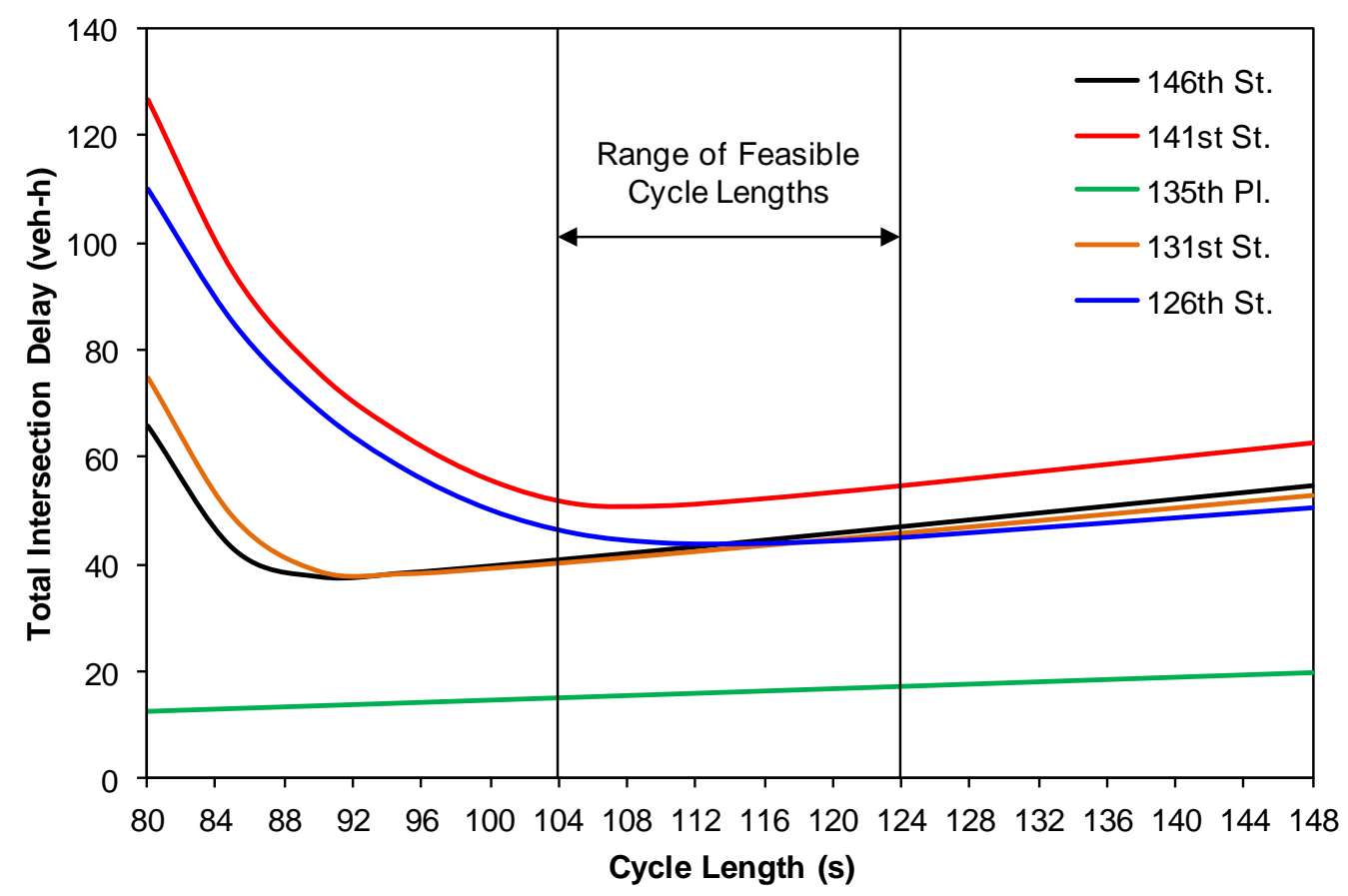

(b) Capacity allocation: Highway Capacity Manual delay curves by intersection.

Figure 2. Model-based analyses of cycle length impact. 
The other aspect of signal operations is capacity allocation. The Highway Capacity Manual delay equation is commonly used to evaluate signalized intersection level of service by movement, approach, and intersection [7]. Figure $2 \mathrm{~b}$ shows a plot of the total delay by intersection predicted by the $H C M$ based on the observed volumes and splits in use on SR 37, for a range of cycle lengths. The results show minimal delay within the software-based feasible range (104 to 124 seconds) for $141^{\text {st }}$ St. and $126^{\text {th }}$ St. (red and blue curves), and monotonically increasing delay for the other three intersections. The minimum delay for $126^{\text {th }}$ St., the most saturated intersection, occurs at 113 seconds. The ideal cycle length from the capacity perspective falls within the same region as that expected for ideal progression.

\section{METHODOLOGY}

From the results of the model analysis, it is hypothesized that actual field performance within the cycle length range of 104 to 124 seconds should yield optimal performance. As recommended by the software and analytical models, a 112 and 113-second cycle should achieve the best progression and allocation of capacity, respectively.

Each intersection was instrumented with inductive loop detectors and signal controllers capable of logging high-resolution event data at 0.1 -second time intervals, including vehicle detection, phase on and off, and phase termination events. Bluetooth sensors were placed at the system endpoints to measure travel times across the corridor, as shown in Figure 1.

The corridor was programmed for actuated-coordinated operation during the study with a $10 \%$ split extension setting on the coordinated phases to allow non-coordinated phases to inherit extra time from the mainline. The actuated-coordinated operation also allows for early mainline returns to green when non-coordinated phases gap out. In the range of 104 to 124 seconds, 11 cycle lengths were tested at the corridor between May $9^{\text {th }}$ and July $24^{\text {th }}$ (Figure 3). This schedule was selected to avoid a monotonic increase or decrease in cycle lengths over the study period.

Offsets were optimized and implemented for each cycle length adjustment to reduce the effect of offsets in the cycle length comparisons. The offset selection process required an initial assessment of the arrival characteristics at the study location using high-resolution controller event data [8]. With the collected data, the Link Pivot progression optimization algorithm predicted a baseline set of offsets to attempt to maximize the percent of vehicles arriving in the green band [9]. The new offsets were then programmed into the controllers and were run on a separate day with the same cycle length. The vehicle arrival and travel time data for the post-optimized day were collected. Each cycle length evaluation consisted of three days of testing and observations (Figure 3). 
Table 1. Study calendar.

\begin{tabular}{|c|c|c|}
\hline Evaluation & Observed Data & Cycle Length (Seconds) \\
\hline Wednesday, May 08, 2013 & Thursday, May 09, 2013 & 104 \\
\hline Monday, June 03, 2013 & Tuesday, June 04, 2013 & 108 \\
\hline Wednesday, May 15, 2013 & Wednesday, May 22, 2013 & 110 \\
\hline Tuesday, May 28, 2013 & Wednesday, May 29, 2013 & 112 \\
\hline Thursday, June 20, 2013 & Tuesday, July 02, 2013 & 116 \\
\hline Tuesday, July 16, 2013 & Wednesday, July 17, 2013 & 118 \\
\hline Thursday, May 30, 2013 & Wednesday, June 19, 2013 & 120 \\
\hline Tuesday, June 11, 2013 & Tuesday, June 18, 2013 & 122 \\
\hline Tuesday, July 23, 2013 & Wednesday, July 24, 2013 & 124 \\
\hline Wednesday, June 05, 2013 & Thursday, June 06, 2013 & \\
\hline Friday, May 10, 2013 & Monday, May 13, 2013 & \\
\hline
\end{tabular}

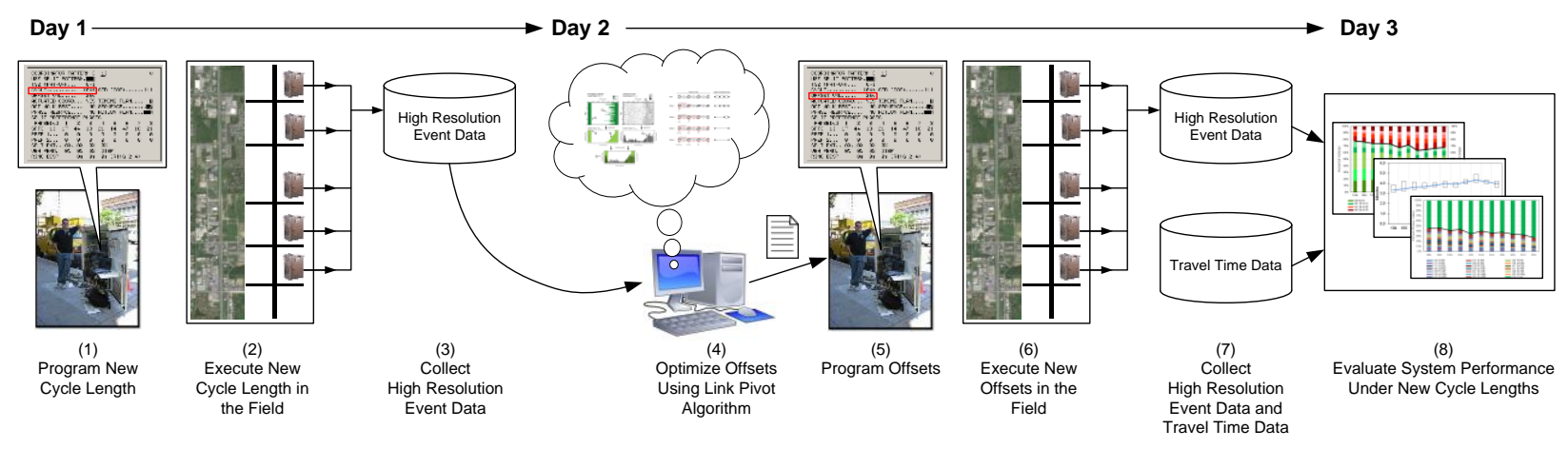

Figure 3. Cycle length evaluation procedure.

- Day 1 (Assessment) - one cycle length was programmed for all five intersections before 19:00. The plan was allowed to run through the study period while the high-resolution data was logged with each intersection controller.

- Day 2 (Observing optimized performance). High-resolution data from Day 1 was used as input for the Link Pivot algorithm (9) to compute a new set of offsets that produced the highest predicted percent of vehicles on green for both directions. If the volumes detected in the controller did not correlate to the other cycle length study period volumes (i.e. higher volumes during a special event) Day 1 was repeated. The new offsets were then programmed into the five controllers before the plan started at 19:00 and was allowed to run through the study period. The cycle length remained unchanged from Day 1.

- Day 3 (Evaluation) - the high-resolution data and Bluetooth travel time data for Day 2 of the study period were collected. If the volumes detected in the controller did not correlate to Day 1 volumes, Day 2 was repeated. 


\section{OBSERVED PERFORMANCE}

The results of the study were evaluated using four performance measures:

(i) Purdue Coordination Diagram (PCD);

(ii) Percent of vehicles arriving on green (POG);

(iii) Travel time;

(iv) Number of force-off phase terminations for side-street through movements.

Items (i), (ii), and (iii) were used to evaluate mainline progression. Item (iv) was used to evaluate how well side-street demand was served at all five intersections.

\section{Mainline Progression}

A combination of PCDs at $141^{\text {st }}$ Street is illustrated for all days of the study period in Figure 4 in increasing cycle length order. The green and red lines mark the beginning of green (BOG) and end of green (EOG), respectively. The graphs qualitatively suggest that the majority of vehicle arrivals at this intersection occur within the green bands for both the northbound and southbound directions for each day of the study. The other four intersections were observed to behave similarly throughout the study (not shown). In this case, the Link Pivot algorithm was able to consistently identify an offset that allowed for good progression for each of the cycle lengths tested. Additionally, it can be seen from the slope of the EOG line that as the length of the cycle increases, the green band increases in proportion. This allowed more vehicles to be accommodated per cycle in the mainline northbound and southbound approaches. However, the figure does not suggest that any particular cycle length was resonant (i.e., performing exceptionally well in comparison to other cycle lengths).

The number of vehicles arriving during red (AOR) or green (AOG) is shown in Figure 5 at intersection for every cycle length tested. The northbound volumes were about $34 \%$ higher than southbound volumes due to regional travel patterns. Overall for the eleven tested cycle lengths, the daily volumes remained within $14 \%$ of the maximum volume in each direction during the 19:00 to 22:00 period on all approaches. Figure 6 illustrates the number of vehicles that arrived during the red and green phase as a percentage of all arrivals at all approaches for each of the cycle length implementations. Both northbound and southbound approaches show an overall decrease in the POG as the cycle length increases for all intersections combined. For the northbound direction, the cycle length with the highest POG was 104 seconds (77.6\%) while the lowest POG was 124 seconds $(67.1 \%)$. For the southbound direction, the cycle length with the highest POG was 104 seconds (77.5\%) while the lowest POG was 118 seconds (63.3\%). On the northbound approaches, the POG varied as much as $11 \%$ from the shorter to the longer cycle lengths. On the southbound approaches, the POG varied as much as $14 \%$. The POG results do not suggest any particular cycle length was resonant within the 104 to 124 second range for this corridor. 


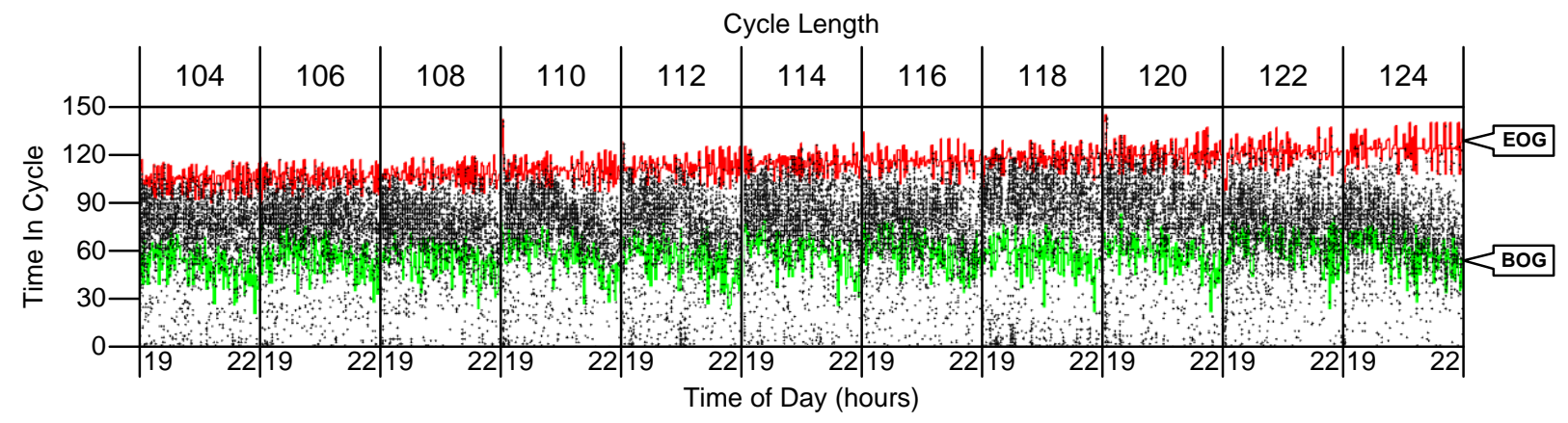

(a) Northbound through movement.

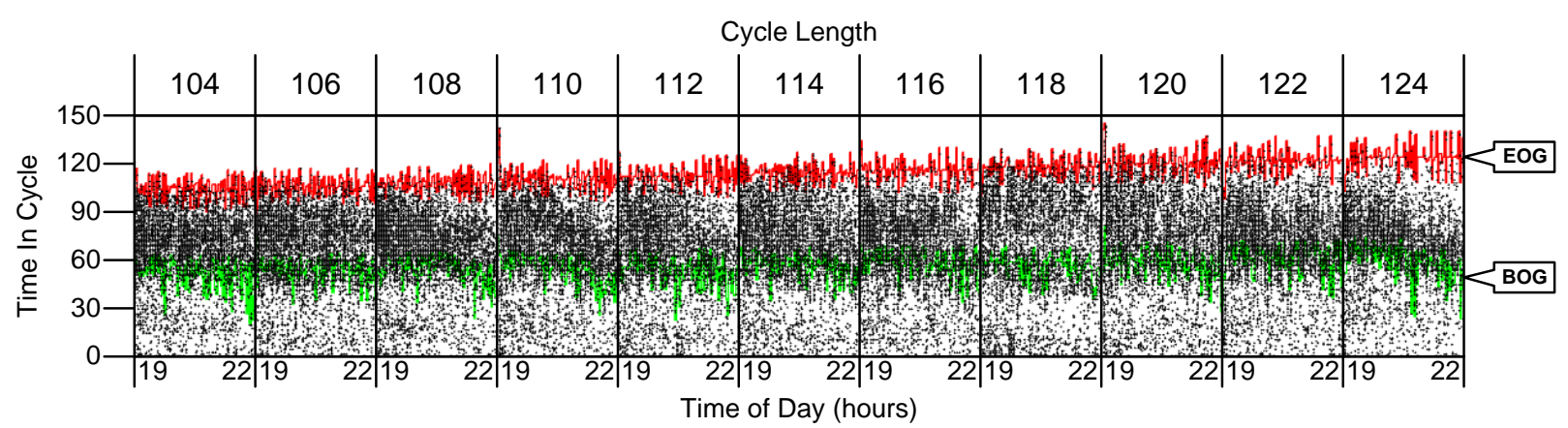

(b) Southbound through movement.

Figure 4. Purdue Coordination Diagram at $141^{\text {st }}$ Street for a range of cycle lengths. 


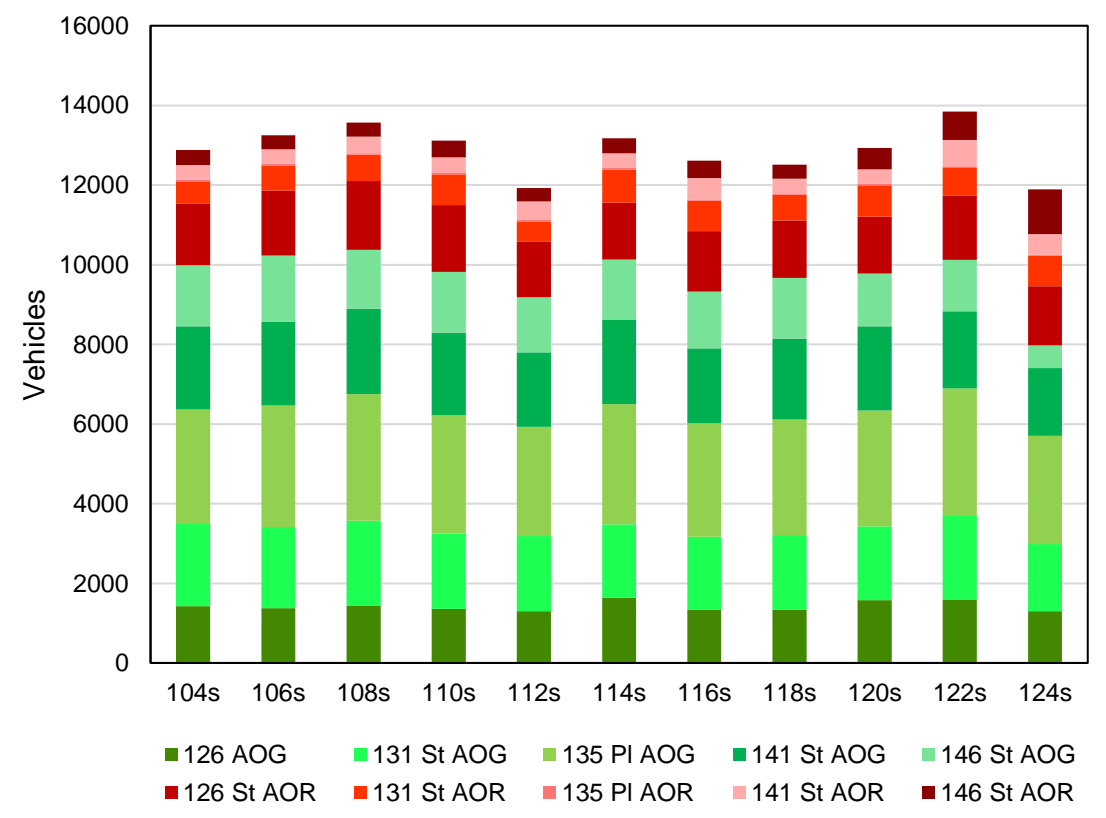

(a) Northbound through arrivals.

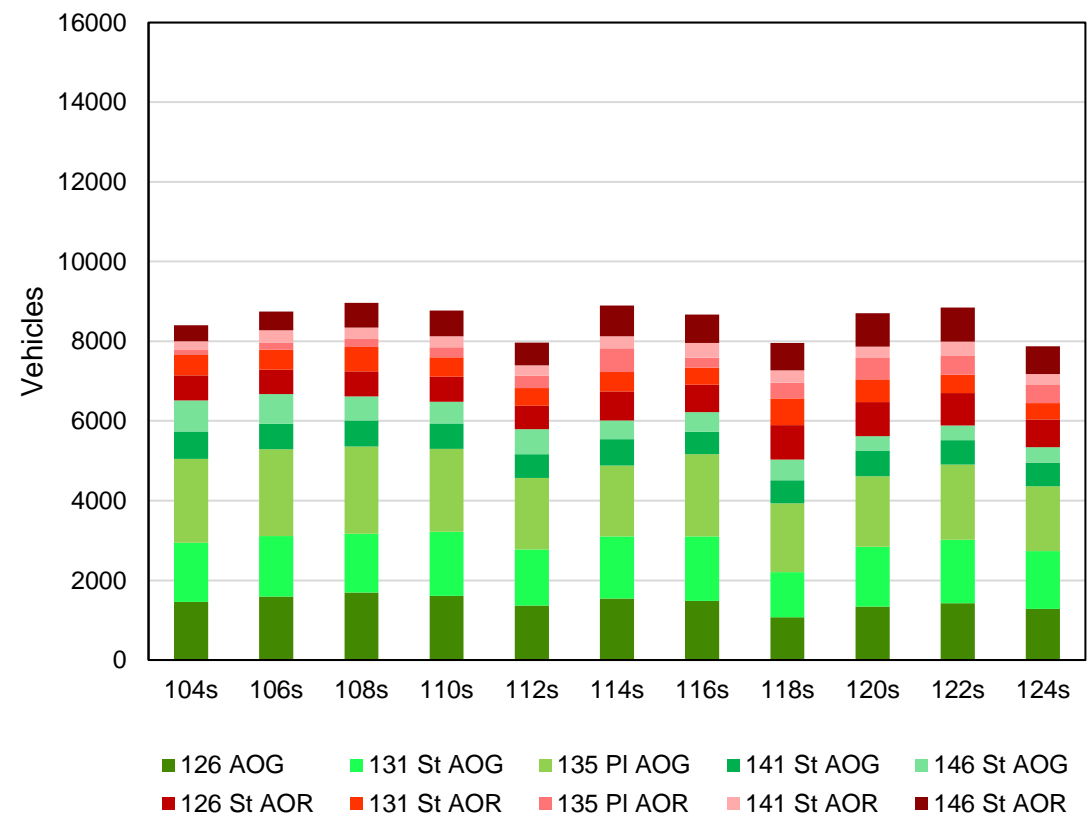

(b) Southbound through arrivals.

Figure 5. Vehicle arrivals for mainline through movement for all intersections. 


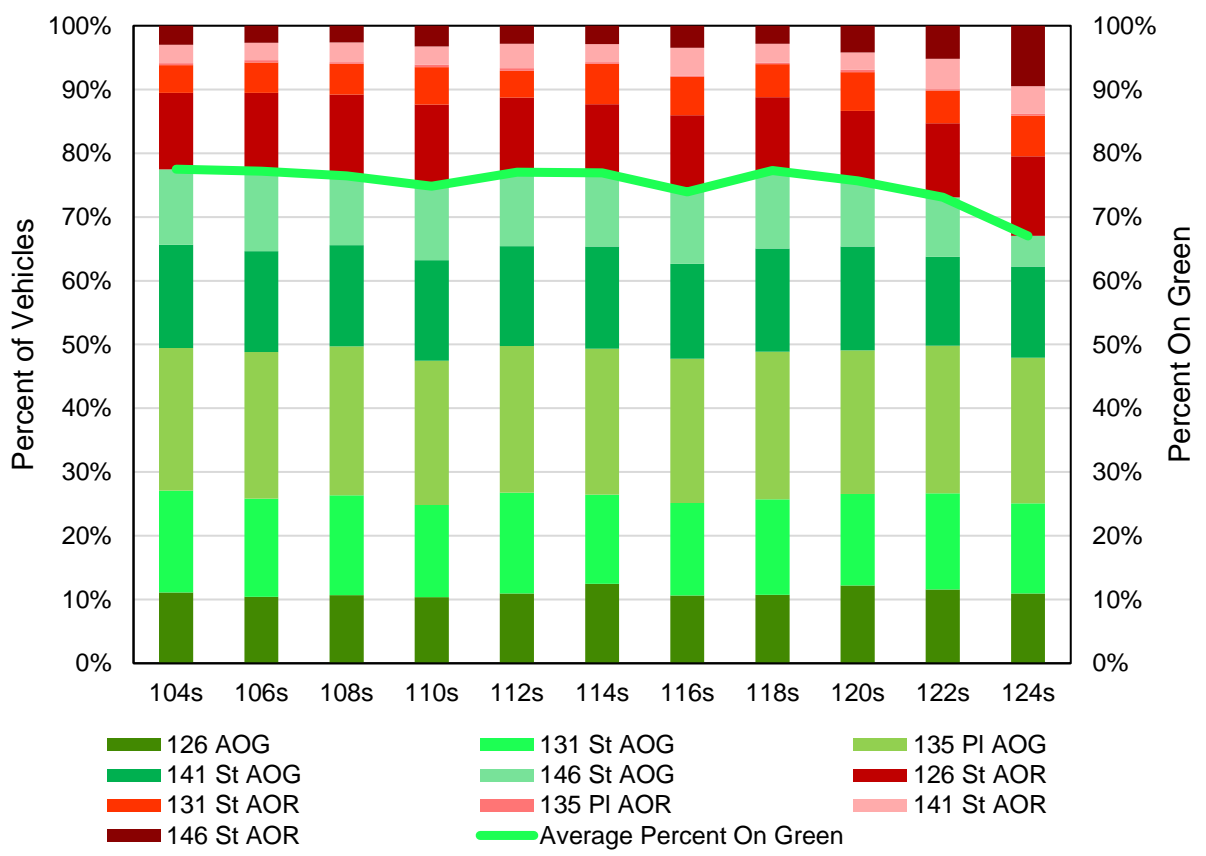

(a) Northbound through arrivals.

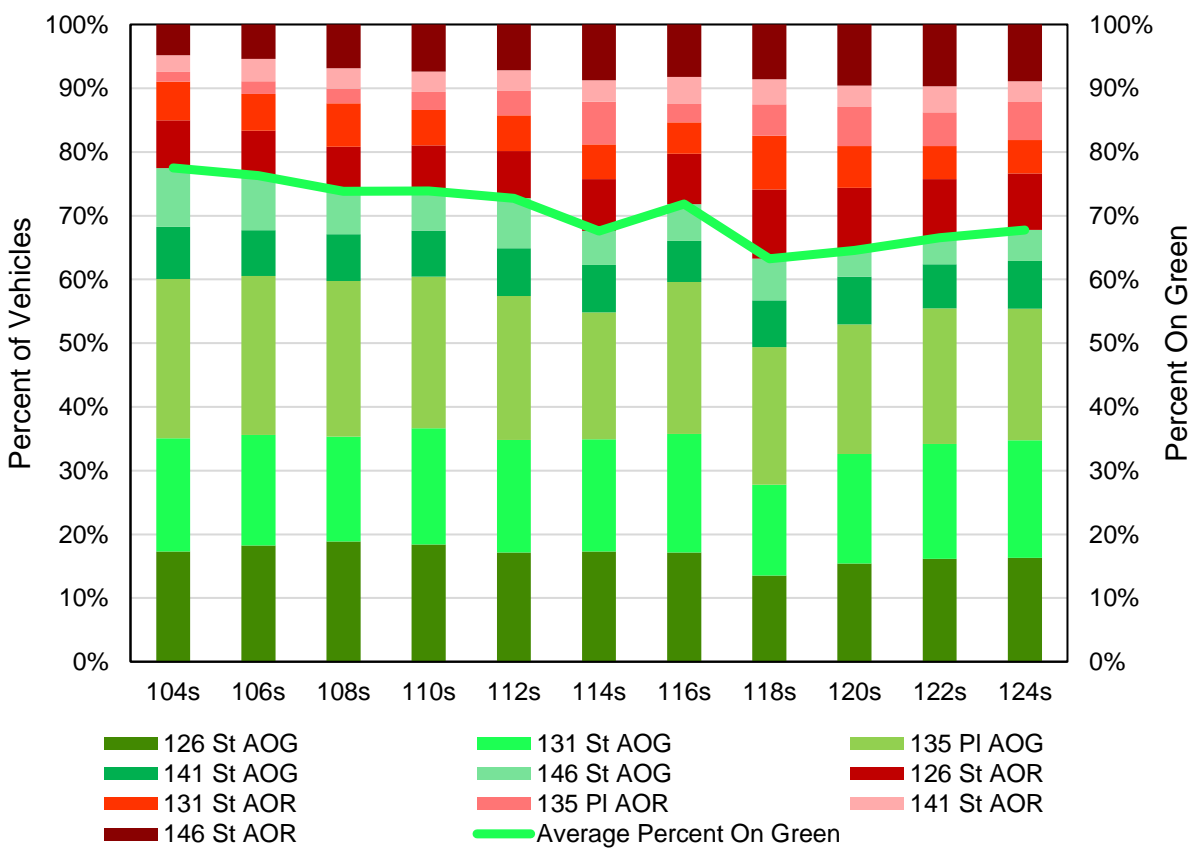

(b) Southbound through arrivals.

Figure 6. Vehicle arrivals for mainline through movement for all intersections, in percentage. 


\section{Travel Time}

Figure 7 shows an inter-quartile distribution of travel times computed from Bluetooth data for each of the tested cycle lengths. The number of samples ranged from 26 to 56 samples per three hour study period per day. The $25^{\text {th }}$ percentile, median, and $75^{\text {th }}$ percentile travel times are represented by the bottom edge of the rectangle, the blue line, and the top edge of the rectangle respectively. The northbound direction observed a minimum median travel time of 3.4 minutes at a 116 second cycle and a maximum of 4.2 minutes at a 124 second cycle. The southbound direction observed the lowest median travel time of 3.3 minutes at a 104 second cycle and the highest travel time of 4.3 minutes at a 120 second cycle. The southbound median travel times overall exhibited a steady increase as cycle length was increased.

\section{Side-Street Performance}

Force off phase termination events were used as an indicator of unserved side-street demand. Figure 8a shows the number of force offs for all side-street phases stacked with the total number of gap outs for those phases. The number of times a phase can be served in the 3-hour study period decreases as cycle length increases (from 103 times for a 104 second cycle to 87 times for a 124 second cycle). Figure $8 \mathrm{~b}$ shows the force offs and gap outs as a percentage of all phases served for the side-street movements. As cycle length increases, the percentage of phases on the side-streets that were terminated with a force off decreased. The percentage of force offs were highest at $46 \%$ for a 106 second cycle and lowest at $27 \%$ for a 124 second cycle. Fewer force-offs may indicate fewer split failures and lower side street delay. 


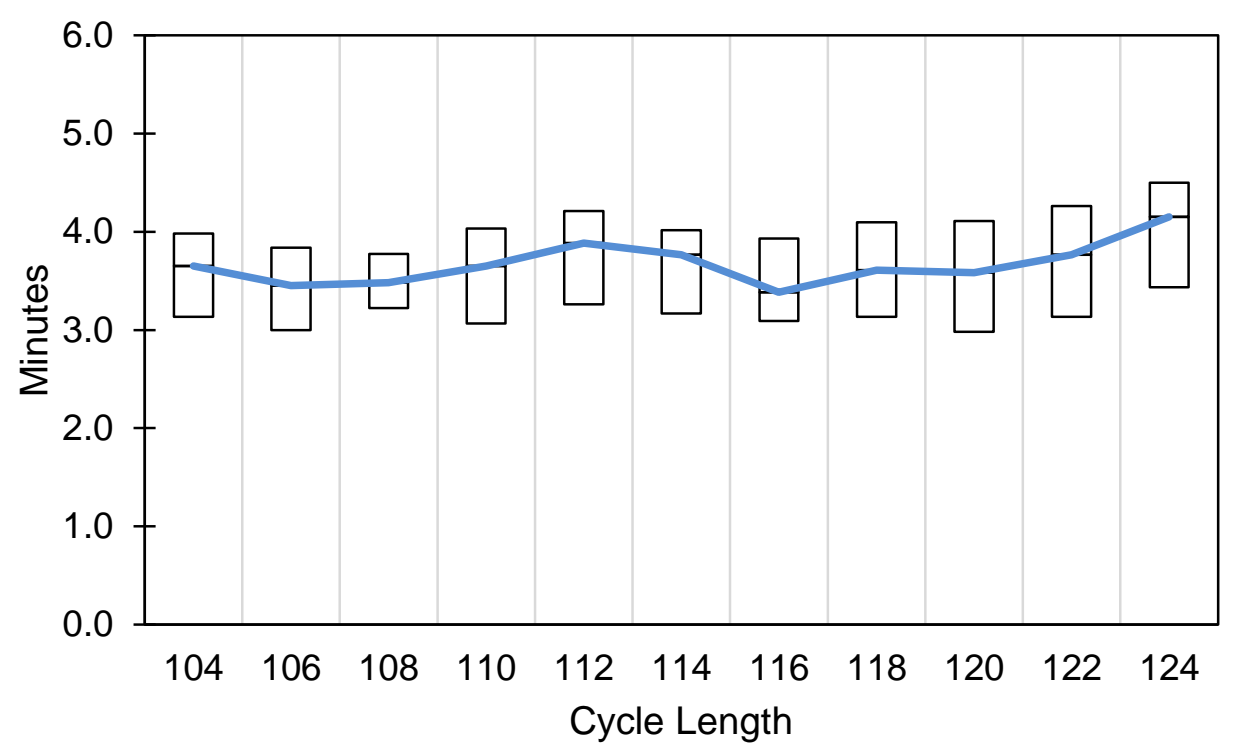

(a) Northbound.

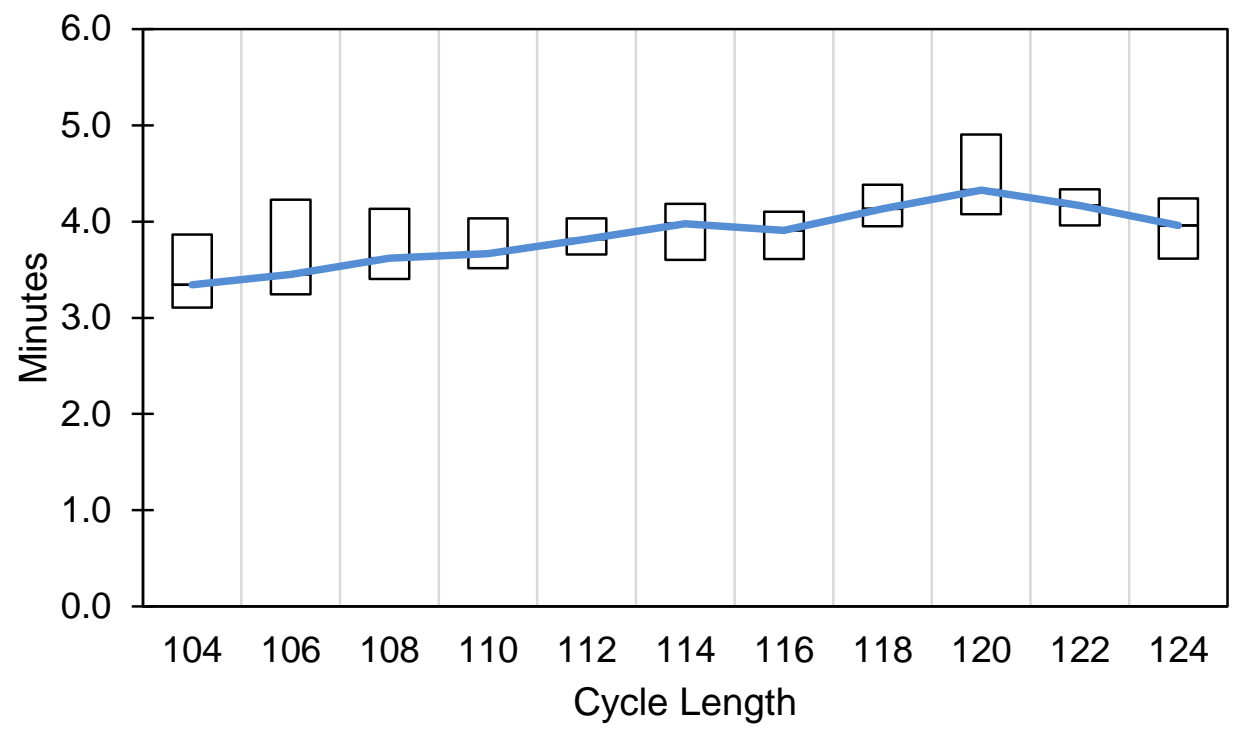

(b) Southbound.

Figure 7. Travel time distribution of study corridor for a range of cycle lengths. 


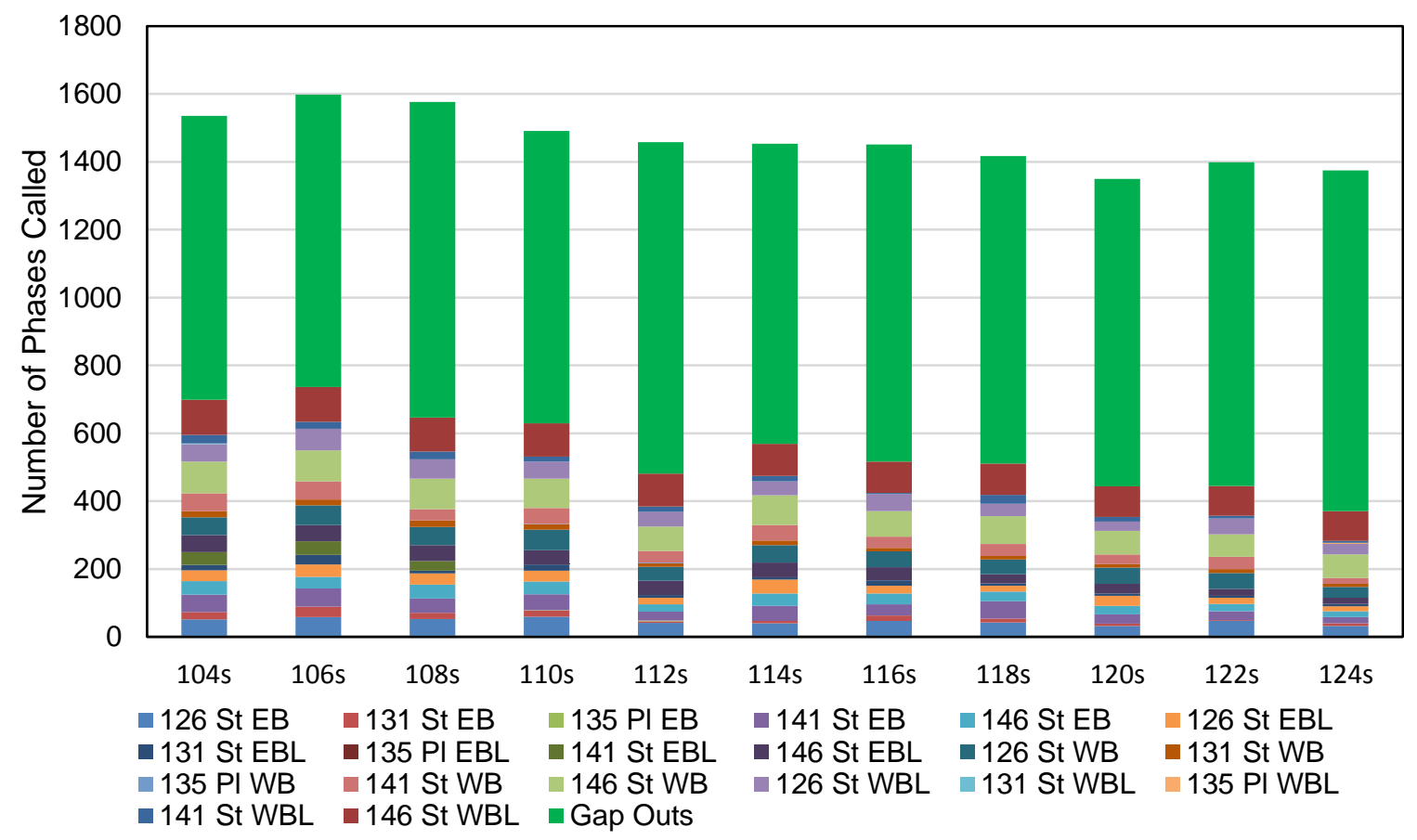

(a) Count of force offs and gap outs.

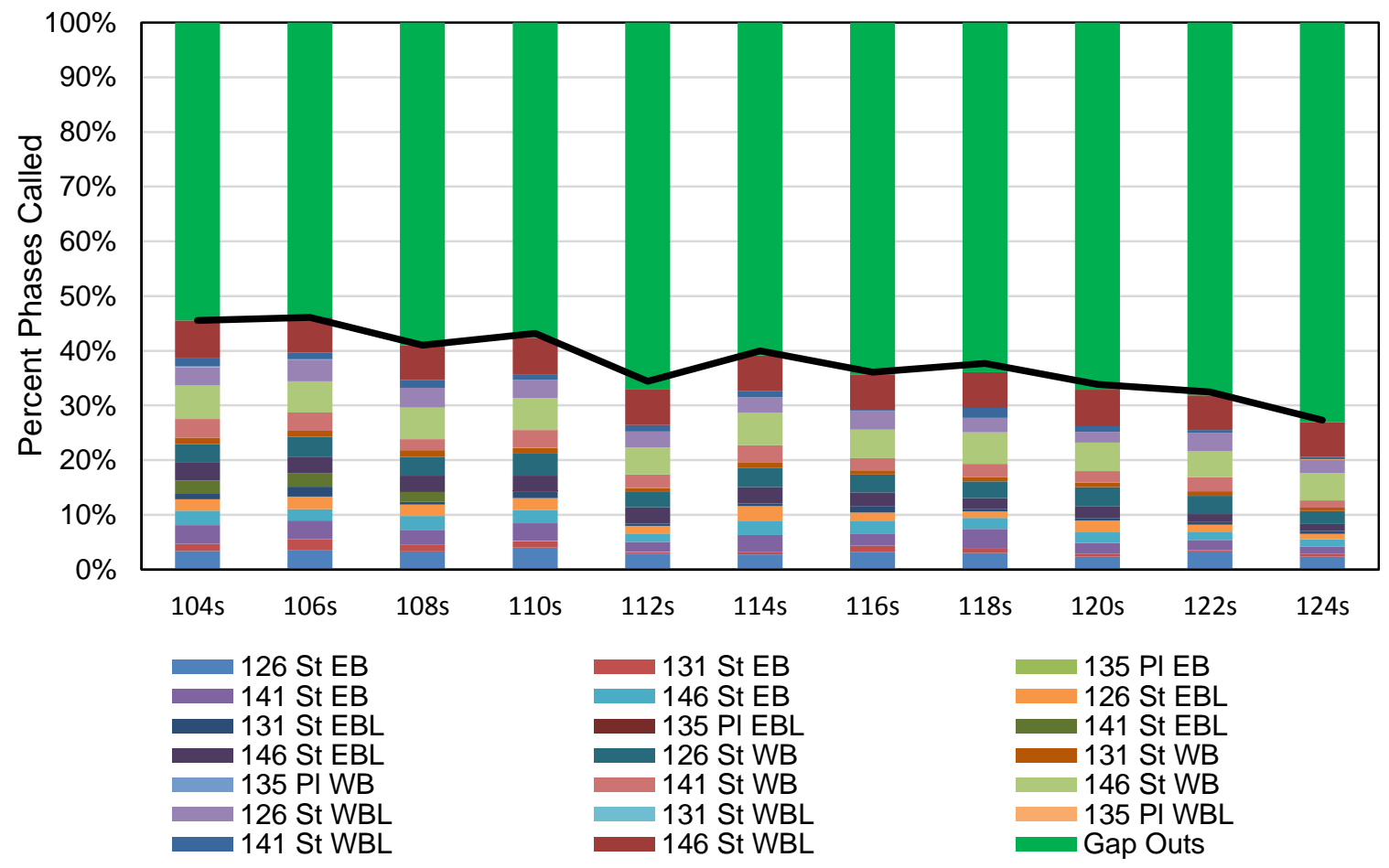

(b) Percentage of force offs and gap outs.

Figure 8. Force offs per phase and total gap outs for side-street approaches. 


\section{SEEMINGLY UNRELATED REGRESSION}

Seemingly Unrelated Regression (SURE) was used to model the northbound/southbound corridor percent arrival on green and corridor travel time and to measure the marginal effects that various parameters contributed. Since both dependent variables (POG and Travel Time) are continuous, linear regression is used. However, SURE assumes that the error terms in the models are correlated [10]; for this reason, general least squares was used to estimate the final models. The SURE model assumption is reasonable as the northbound and southbound travel times during the evaluation period would likely have correlated error terms. Using the information from the models can help measure the amount of impact that each of the significant parameters has on the variable of interest. The parameter estimates are presented in Table 2 and explained subsequently.

Table 2. Parameter estimates for seemingly unrelated regression

\begin{tabular}{|c|c|c|c|c|c|}
\hline \multirow[b]{2}{*}{ Variable } & \multirow[b]{2}{*}{$\begin{array}{l}\text { Average } \\
\text { (Std.Dev) }\end{array}$} & \multicolumn{2}{|c|}{$\begin{array}{l}\text { Seemingly Unrelated Regression of } \\
\text { Corridor Percent on Green }\end{array}$} & \multicolumn{2}{|c|}{$\begin{array}{c}\text { Seemingly Unrelated Regression of } \\
\text { Corridor Travel Time (Minutes) }\end{array}$} \\
\hline & & $\begin{array}{c}\text { Northbound } \\
\text { (t-statistic) }\end{array}$ & $\begin{array}{c}\text { Southbound } \\
(\mathrm{t} \text {-statistic) }\end{array}$ & $\begin{array}{c}\text { Northbound } \\
\text { (t-statistic) }\end{array}$ & $\begin{array}{c}\begin{array}{c}\text { Southbound } \\
\text { (t-statistic) }\end{array} \\
\end{array}$ \\
\hline Constant & & $\begin{array}{c}1.22 \\
(10.76)\end{array}$ & $\begin{array}{c}1.88 \\
(9.03)\end{array}$ & $\begin{array}{c}4.44 \\
(2.83)\end{array}$ & $\begin{array}{l}4.07 \\
(2.81)\end{array}$ \\
\hline Cycle Length (Seconds) & $\begin{array}{c}114 \\
(6.64)\end{array}$ & $\begin{array}{c}-0.0023 \\
(-2.31)\end{array}$ & $\begin{array}{c}-0.0075 \\
(-4.07)\end{array}$ & $\begin{array}{l}0.012 \\
(1.52)\end{array}$ & $\begin{array}{l}0.017 \\
(2.61)\end{array}$ \\
\hline Percent Side Street Force Off & $\begin{array}{c}0.38 \\
(0.058)\end{array}$ & -- & $\begin{array}{l}-0.39 \\
(-2.71)\end{array}$ & -- & -- \\
\hline Volume (NB), in thousands & $\begin{array}{c}12,884 \\
(616)\end{array}$ & -- & -- & $\begin{array}{l}-0.17 \\
(-1.98)\end{array}$ & -- \\
\hline Volume (SB), in thousands & $\begin{array}{l}8,528 \\
(409)\end{array}$ & -- & -- & -- & $\begin{array}{l}0.090 \\
(1.58)\end{array}$ \\
\hline Percent on Green (NB) & $\begin{array}{c}0.75 \\
(0.031)\end{array}$ & N/A & N/A & -- & -- \\
\hline Percent on Green (SB) & $\begin{array}{c}0.71 \\
(0.048)\end{array}$ & N/A & N/A & -- & $\begin{array}{l}-4.15 \\
(-4.81)\end{array}$ \\
\hline Travel Time (NB) (Minutes) & $\begin{array}{c}3.67 \\
(0.22)\end{array}$ & $\begin{array}{l}-0.055 \\
(-2.47)\end{array}$ & -- & N/A & N/A \\
\hline Travel Time (SB) (Minutes) & $\begin{array}{c}3.85 \\
(0.31)\end{array}$ & -- & $\begin{array}{l}-0.044 \\
(-1.79)\end{array}$ & N/A & N/A \\
\hline Adjusted $\mathrm{R}^{2}$ & & 0.46 & 0.83 & 0.23 & 0.91 \\
\hline Log Likelihood & & 29.59 & 32.57 & 6.17 & 15.78 \\
\hline Number of Observations & & 11 & 11 & 11 & 11 \\
\hline Average Prediction Error & & $0.6 \%$ & $-1.0 \%$ & $-1.3 \%$ & $-0.4 \%$ \\
\hline
\end{tabular}

-- indicates not used in the model but included for descriptive statistics 


\section{SURE - Percent on Green}

The first SURE model estimated the percent on green for both the northbound and southbound directions and utilized their correlated error terms to help achieve efficient parameter estimates by leveraging the additional information in the residuals. The equations estimated for the corridor percent on green (POG) are shown below in Equation 2 and Equation 3:

$$
\begin{aligned}
& P O G_{N B}=1.22-0.0023 *(\text { Cycle Length })-0.055 *\left(T T_{N B}\right) \quad \text { Equation 2 } \\
& P O G_{S B}=1.87-0.0075 *(\text { Cycle Length })-0.044 *\left(T T_{S B}\right)-0.39 \quad \text { Equation } 3 \\
& *(\% \text { SSFO })
\end{aligned}
$$

Where:

$P O G_{N B}$ represents the northbound percent on green, $P O G_{S B}$ represents the southbound percent on green,

Cycle Length represents the programmed cycle length for the corridor in seconds, $T T_{N B}$ represents the northbound corridor travel time in minutes, $T T_{S B}$ represents the southbound corridor travel time in minutes, and $\% S S F O$ represents the percent of forced-off side-street phases for the corridor.

For both the northbound and southbound models, an increase in cycle length was estimated to cause a decrease in the percent on green. A 1-second increase in cycle length resulted in a predicted decrease of the northbound POG by $0.23 \%$ and the southbound POG by $0.75 \%$. This is consistent with the trends shown in Figure 6. For the northbound direction, a measured increase in travel time suggested a decrease in the POG. For each minute of increase, a 5.5\% reduction in percent on green is expected. This makes sense, as slow traffic may represent poor progression, which would result in fewer vehicles arriving in the green band. For the southbound equation, a decrease in the percent of force offs on the side street was estimated to decrease the percent on green on the southbound main line. If the mainline forces off, the time is returned to the mainline where the platoon would be progressed to the next red and thus reduce the percent of traffic arriving on green. And similar to the northbound direction, an increase in southbound corridor travel time estimated a decrease in the percent on green. Again, this is likely indicative of poor progression through the corridor where fewer vehicles are arriving on green. 


\section{SURE - Corridor Travel Time}

In a separate model, the northbound and southbound corridor travel times were estimated using SURE. Since travel times for both the northbound and southbound directions were collected at simultaneously, the error terms likely share correlation due to similar unobserved characteristics and thus gave more efficient parameter estimates. The equations are shown below in Equation 4 and Equation 5:

$$
\begin{aligned}
& T T_{N B}=4.44+0.012 *(\text { Cycle Length })-0.17 *\left(\text { Volume }_{N B}\right) \quad \text { Equation } 4 \\
& T T_{S B}=4.07+0.017 *(\text { Cycle Length })+0.090 *\left(\text { Volume }_{S B}\right)-4.17 \quad \text { Equation } 5 \\
& *\left(P O G_{S B}\right)
\end{aligned}
$$

Where:

$T T_{N B}$ represents the northbound corridor travel time in minutes,

$T T_{S B}$ represents the southbound corridor travel time in minutes,

Cycle Length represents the programmed cycle length for the corridor in seconds,

Volume $_{N B}$ represents the northbound volume in thousands of vehicles,

Volume $_{S B}$ represents the southbound volume in thousands of vehicles,

and $P O G_{S B}$ represents the southbound percent on green.

For both the northbound and southbound models, an increase in the cycle length indicated an increase in the corridor travel time. A 1-second increase in the cycle length estimated a 0.012 minute $(0.72$ seconds) increase in the northbound corridor travel time and a 0.017 minute (1.02 seconds) increase in the southbound corridor travel time. This is consistent with trends shown in Figure 7. For the northbound direction, an increase in traffic volume resulted in a decrease in northbound corridor travel time. This may seem counter-intuitive at first, but because this experiment was controlled within a consistent range of volumes, the results would only apply for the volumes observed. What is likely happening is that a slight increase in traffic is keeping the extension timers active and thus reducing any gap outs on the heavier mainline actuatedcoordinated movement. In the southbound direction, an increase in volume caused an increase in travel time. Since the link pivot algorithm tends to favor the direction with heavier traffic, an increase in the southbound traffic likely would experience a more intuitive increase in travel time based on congestion (and thus phase extension would not a benefit gained from increased traffic). Finally, an increase in percent on green in the southbound direction was estimated to decrease the southbound corridor travel time. This makes sense as good progression would be expected to contribute to reduced travel times. 


\section{Overall Fit}

The southbound achieved very good overall fit based on the adjusted- $\mathrm{R}^{2}$ values. From Table 2:

- The northbound POG model achieved a reasonable adjusted- $\mathrm{R}^{2}$ of 0.46 with an average prediction error of $0.6 \%$ (Figure 9a)

- The southbound POG model achieved a good adjusted- $\mathrm{R}^{2}$ of 0.83 with an average prediction error of $-1.0 \%$ (Figure $9 b$ )

- The northbound travel time model achieved a reasonable adjusted-R $\mathrm{R}^{2}$ of 0.23 with an average prediction error of $-1.3 \%$ (Figure 9c)

- The southbound travel time model achieved a good adjusted- $\mathrm{R}^{2}$ of 0.91 with an average prediction error of $-0.4 \%$ (Figure $9 \mathrm{~d}$ )

In the graphs, it is clear that the southbound models (Figure 9b and Figure 9d) achieved exceptional fit while the northbound models (Figure 9a and Figure 9c) would have likely benefited from more data spanning a broader range of values.

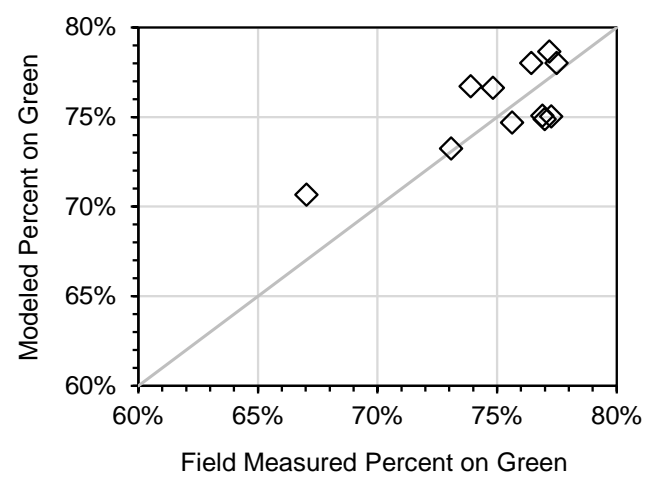

a) Northbound percent on green

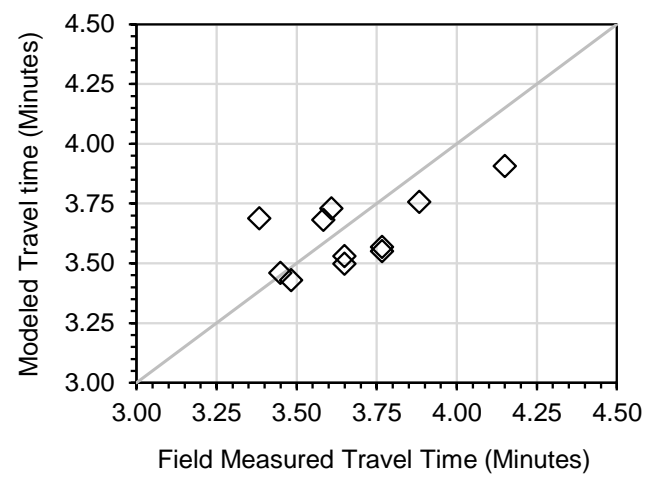

c) Northbound travel time

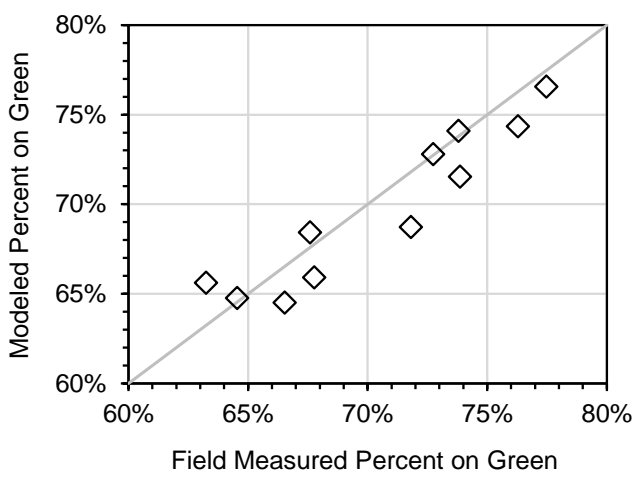

b) Southbound percent on green

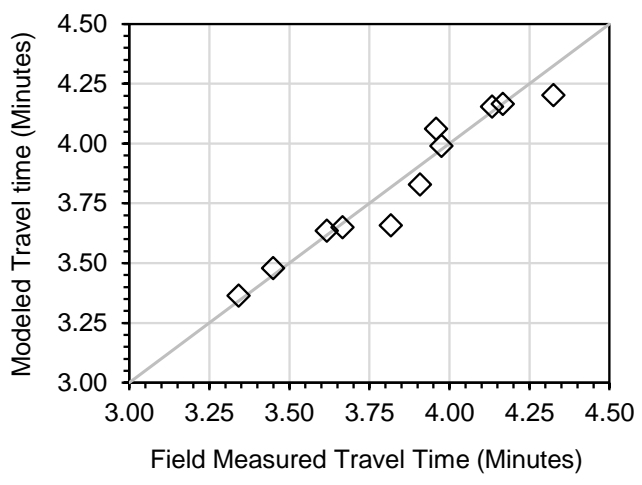

d) Southbound travel time

Figure 9. Comparison of empirical measurements and modeled predictions 


\section{CONCLUSIONS}

A set of 11 cycle lengths were tested on a 5-intersection corridor and the performance results were presented. Model results suggested that a cycle length of 112 to 113 seconds would provide optimal performance, but also suggested that a range of cycle lengths ranging from 104 seconds to 124 seconds would be feasible. No conclusive evidence of a resonant cycle was found by the experiments within that range. Although it is possible that a cycle length beyond the range explored in this study might have exhibited resonant behavior, cycle lengths less than 104 seconds or greater than 124 seconds were not considered appropriate for the test corridor.

- The percentage of vehicles arriving on green (POG) decreased as cycle length increased for both northbound and southbound directions. However, the smaller POG did not increase travel times northbound as substantially as in the southbound direction most likely because the higher northbound volume was inherently favored by the progression optimization.

- The decrease in the number of force offs for the side-street movements as cycle length was increased may correspond to an overall delay tradeoff (from a reduction in split failures) when using longer cycles.

- The SURE method was used to evaluate the correlation between cycle length, percent on green, and travel time. The model results were consistent with empirical data as there was an inverse relationship between cycle length and percent of vehicles arriving on green, and also a direct relationship between cycle length and corridor travel times. The model also suggested that Link Pivot optimization was able to maintain relatively consistent performance across the different cycle lengths even though the general trend was decreasing performance (increasing travel time and decreasing POG) as cycle length increased.

In summary, the results found that, within a 20 -second range of feasible cycle lengths identified by software and analytical models, a particular cycle length with substantially different performance did not emerge. Instead, the trends in the performance measures examined (arrivals on green, travel time, and number of force-offs) were rather monotonic with respect to cycle length. The findings suggest that a resonance-based cycle length selection policy may not yield superior performance compared to the more traditional policy of providing a modestly short cycles with reserve capacity to accommodate stochastic variation in traffic volumes. 


\section{ACKNOWLEDGEMENT}

This work was supported by the Indiana Department of Transportation. The contents of this paper reflect the views of the authors, who are responsible for the facts and the accuracy of the data presented herein, and do not necessarily reflect the official views or policies of the sponsoring organizations. These contents do not constitute a standard, specification, or regulation.

\section{REFERENCES}

1. Shelby, S.G., D.M. Bullock, and D. Gettman. "Resonant Cycles in Traffic Signal Control." Transportation Research Record No. 1925, pp. 215-226, 2005.

2. Robertson, D.I. and R.D. Bretherton. "Optimizing Networks of Traffic Signals in Real Time-The SCOOT Method.” IEEE Transactions on Vehicular Technology, Vol. 40, pp. 11-15, 1991.

3. Gartner, N.H., F.J. Pooran, and C.M. Andrews. "Optimized Policies for Adaptive Control Strategy in Real-Time Traffic Adaptive Control Systems.” Transportation Research Record No. 1811, pp. 148-156, 2002.

4. Koshi, M. "Cycle Time Optimization in Traffic Signal Coordination.” Transportation Research, Part A, Vol. 23, pp. 29-34, 1989.

5. Ladron de Guevara, F., M.D. Hickman, and L. Head. "Evaluation of Resonant Cycles.” Transportation Research Board Annual Meeting, Paper No. 09-1924, 2009.

6. Synchro 8.0 User's Guide. Trafficware, Sugar Land, Texas, 2011.

7. Highway Capacity Manual. TRB, National Research Council, Washington, D.C., 2010.

8. Smaglik, E.J., A. Sharma, D.M. Bullock, J.R. Sturdevant, and G. Duncan. "Event-Based Data Collection for Generating Actuated Controller Performance Measures." Transportation Research Record No. 2035, Transportation Research Board of the National Academies, Washington, DC, pp. 97-106, 2007.

9. Day, C.M. and D.M. Bullock, "Computational Efficiency of Alternative Algorithms for Arterial Offset Optimization," Transportation Research Record: Journal of the Transportation Research Board, No. 2259, Transportation Research Board of the National Academies, Washington, D.C., pp. 37-47, 2011. DOI: 10.3141/2259-04.

10. Washington, S.P., M.G. Karlaftis, F.L. Mannering, "Statistical and Econometric Methods for Transportation Data Analysis", CRC Press. Boca Raton. Pp 155-156. 2011. 